\title{
Open
}

\section{Phenotype and genotype of 87 patients with Mowat-Wilson syndrome and recommendations for care}

Ivan Ivanovski, MD ${ }^{1,2}$, Olivera Djuric, MD, MSc ${ }^{1,3}$, Stefano Giuseppe Caraffi, PhD ${ }^{1}$, Daniela Santodirocco, MD ${ }^{1}$, Marzia Pollazzon, $\mathrm{MD}^{1}$, Simonetta Rosato, MD ${ }^{1}$, Duccio Maria Cordelli, MD ${ }^{4}$, Ebtesam Abdalla, MD, $\mathrm{PhD}^{5}$, Patrizia Accorsi, MD ${ }^{6}$, Margaret P. Adam, MD, FACMG ${ }^{7}$, Paola Francesca Ajmone, $\mathrm{MD}^{8}$,

Magdalena Badura-Stronka, $\mathrm{MD}, \mathrm{PhD}^{9}$, Chiara Baldo, $\mathrm{PhD}^{10}$, Maddalena Baldi, $\mathrm{MSc}^{1}$, Allan Bayat, MD ${ }^{11,12}$, Stefania Bigoni, MD ${ }^{13}$, Federico Bonvicini, MD ${ }^{1,14}$, Jeroen Breckpot, MD ${ }^{15}$,

Bert Callewaert, MD, $\mathrm{PhD}^{16}$, Guido Cocchi, MD ${ }^{17}$, Goran Cuturilo, MD, $\mathrm{PhD}^{18,19}$,

Daniele De Brasi, MD ${ }^{20}$, Koenraad Devriendt MD, PhD ${ }^{15}$, Mary Beth Dinulos, MD ${ }^{21}$,

Tina Duelund Hjortshøj, MD, PhD ${ }^{22}$, Roberta Epifanio, MD ${ }^{23}$, Francesca Faravelli, MD, MSc ${ }^{24}$, Agata Fiumara, MD ${ }^{25}$, Debora Formisano, MSc ${ }^{26}$, Lucio Giordano, MD ${ }^{6}$, Marina Grasso, PhD ${ }^{10}$,

Sabine Grønborg, MD ${ }^{27}$, Alessandro lodice, $\mathrm{MD}^{28}$, Lorenzo lughetti, MD, $\mathrm{PhD}^{14}$,

Vladimir Kuburovic, MD, PhD ${ }^{29}$, Anna Kutkowska-Kazmierczak, MD, PhD ${ }^{30}$, Didier Lacombe, MD ${ }^{31,32}$, Caterina Lo Rizzo, MD ${ }^{33}$, Anna Luchetti, MD ${ }^{34}$, Baris Malbora, MD ${ }^{35}$, Isabella Mammi, MD ${ }^{36}$,

Francesca Mari, MD, PhD ${ }^{33}$, Giulia Montorsi, MD ${ }^{1,37}$, Sebastien Moutton, $\mathrm{MD}^{31,32}$,

Rikke S. Møller, MD, PhD ${ }^{38,39}$, Petra Muschke, MD ${ }^{40}$, Jens Erik Klint Nielsen, $\mathrm{MD}^{41}$,

Ewa Obersztyn, MD, PhD ${ }^{30}$, Chiara Pantaleoni, MD ${ }^{42}$, Alessandro Pellicciari, $\mathrm{MD}^{4}$,

Maria Antonietta Pisanti, MD ${ }^{43}$, Igor Prpic, MD, PhD ${ }^{44}$, Maria Luisa Poch-Olive, $\mathrm{MD}^{45}$,

Federico Raviglione, $\mathrm{MD}^{46}$, Alessandra Renieri, MD, $\mathrm{PhD}^{33}$, Emilia Ricci, MD ${ }^{4}$, Francesca Rivieri, $\mathrm{MD}^{47}$, Gijs W. Santen, $\mathrm{MD}^{48}$, Salvatore Savasta, MD ${ }^{49}$, Gioacchino Scarano, MD ${ }^{50}$, Ina Schanze, MD ${ }^{40}$,

Angelo Selicorni, MD ${ }^{51,52}$, Margherita Silengo, $\mathrm{MD}^{53}$, Robert Smigiel, MD, $\mathrm{PhD}^{54}$, Luigina Spaccini, MD ${ }^{55}$, Giovanni Sorge, MD ${ }^{56}$, Krzysztof Szczaluba, MD, PhD ${ }^{57}$, Luigi Tarani, MD ${ }^{58}$,

Luis Gonzaga Tone, MD ${ }^{59}$, Annick Toutain, MD ${ }^{60}$, Aurelien Trimouille, MD ${ }^{31,32}$,

Elvis Terci Valera, MD ${ }^{59}$, Samantha Schrier Vergano, MD ${ }^{61,62}$, Nicoletta Zanotta, MD ${ }^{24}$, Martin Zenker, MD ${ }^{40}$, Andrea Conidi, PhD ${ }^{63}$, Marcella Zollino, MD ${ }^{64}$, Anita Rauch, MD ${ }^{65}$, Christiane Zweier, MD ${ }^{66}$ and Livia Garavelli, MD ${ }^{1}$

\footnotetext{
${ }^{1}$ Clinical Genetics Unit, Department of Obstetrics and Pediatrics, AUSL-IRCCS of Reggio Emilia, Reggio Emilia, Italy; ${ }^{2}$ Department of Surgical, Medical, Dental, and Morphological Sciences with Interest in Transplant, Oncology and Regenerative Medicine, University of Modena and Reggio Emilia, Modena, Italy; ${ }^{3}$ Institute of Epidemiology, School of Medicine, University of Belgrade, Belgrade, Serbia; ${ }^{4}$ Child Neurology and Psychiatry Unit, S Orsola Malpighi Hospital, University of Bologna, Bologna, Italy; ${ }^{5}$ Department of Human Genetics, Medical Research Institute, University of Alexandria, Alexandria, Egypt; ${ }^{6}$ Neuropsychiatric Department, Spedali Civili Brescia, Brescia, Italy; ${ }^{7}$ Division of Genetic Medicine, University of Washington School of Medicine, Seattle, Washington, USA; ${ }^{8}$ Child and Adolescent Neuropsychiatric Service (UONPIA) Fondazione IRCCS Ca' Granda Ospedale Maggiore Policlinico, Milan, Italy; ${ }^{9}$ Chair and Department of Medical Genetics, Poznan University of Medical Sciences, Poznań, Poland; ${ }^{10}$ Laboratory of Human Genetics; Galliera Hospital, Genoa, Italy; ${ }^{11}$ Department of Pediatrics, University Hospital of Copenhagen/Hvidovre, Copenhagen, Denmark; ${ }^{12}$ Department of Clinical Genetics, Rigshospitalet, University Hospital of Copenhagen, Copenhagen, Denmark; ${ }^{13}$ UOL of Medical Genetics, University Hospital of Ferrara, Ferrara, Italy; ${ }^{14}$ Pediatric Unit, Department of Medical and Surgical Sciences for Mothers, Children and Adults, University of Modena and Reggio Emilia, Modena, Italy; ${ }^{15}$ Center for Human Genetics, Catholic University of Leuven, Leuven, Belgium; ${ }^{16}$ Center for Medical Genetics, Ghent University Hospital, Ghent, Belgium; ${ }^{17}$ Neonatology Unit, S. Orsola-Malpighi Hospital, University of Bologna, Bologna, Italy; ${ }^{18}$ Faculty of Medicine, University of Belgrade, Belgrade, Serbia; ${ }^{19}$ Department of Medical Genetics, University Children's Hospital, Belgrade, Serbia; ${ }^{20}$ Department of Pediatrics, AORN Santobono Pausilipon, Naples, Italy; ${ }^{21}$ Department of Pediatrics, Dartmouth-Hitchcock Medical Center, Lebanon, New Hampshire, USA; ${ }^{22}$ Department of Clinical Genetics, Kennedy Center, Glostrup, Denmark; ${ }^{23}$ Clinical Neurophysiology Unit, IRCCS, E Medea Scientific Institute, Bosisio Parini, Lecco, Italy; ${ }^{24}$ Clinical Genetics, NE Thames Regional Genetics Service, Great Ormond Street Hospital for Children NHS Foundation Trust, London, UK; ${ }^{25}$ Department of Clinical and Experimental Medicine, Regional Referral Center for Inborn Errors Metabolism, Pediatric Clinic, University of Catania, Catania, Italy; ${ }^{26}$ Scientific Directorate, Arcispedale Santa Maria Nuova-IRCCS, Reggio Emilia, Italy; ${ }^{27}$ Center for Rare Diseases, Department of Clinical Genetics, University Hospital Copenhagen, Copenhagen, Denmark; ${ }^{28}$ Neuropsychiatric Department, Arcispedale Santa Maria NuovaIRCCS, Reggio Emilia, Italy; ${ }^{29}$ Department of Cardiology, Mother and Child Health Care Institute, Belgrade, Serbia; ${ }^{30}$ Department of Medical Genetics, Institute of the Mother and Child, Warsaw, Poland; ${ }^{31}$ CHU de Bordeaux, Service de Génétique Médicale, Bordeaux, France; ${ }^{32}$ INSERM U1211, Univ. Bordeaux, Bordeaux, France; ${ }^{33}$ Medical Genetics, University of Siena, Siena, Italy; ${ }^{34}$ Child Neuropsychiatry Unit, Faculty of Medicine and Psychology, Sapienza University, Rome, Italy; ${ }^{35}$ Department of Pediatric Hematology \& Oncology, Tepecik Training and Research Hospital, Izmir, Turkey; ${ }^{36}$ Medical Genetics Unit, Dolo Hospital, Venice, Italy; ${ }^{37}$ Faculty of Medicine, University of Modena and Reggio
} 
Purpose: Mowat-Wilson syndrome (MWS) is a rare intellectual disability/multiple congenital anomalies syndrome caused by heterozygous mutation of the $Z E B 2$ gene. It is generally underestimated because its rarity and phenotypic variability sometimes make it difficult to recognize. Here, we aimed to better delineate the phenotype, natural history, and genotype-phenotype correlations of MWS.

Methods: In a collaborative study, we analyzed clinical data for 87 patients with molecularly confirmed diagnosis. We described the prevalence of all clinical aspects, including attainment of neurodevelopmental milestones, and compared the data with the various types of underlying $Z E B 2$ pathogenic variations.

Results: All anthropometric, somatic, and behavioral features reported here outline a variable but highly consistent phenotype. By presenting the most comprehensive evaluation of MWS to date, we define its clinical evolution occurring with age and derive suggestions for patient management. Furthermore, we observe that its severity correlates with the kind of $Z E B 2$ variation involved, ranging from $Z E B 2$ locus deletions, associated with severe phenotypes, to rare nonmissense intragenic mutations predicted to preserve some ZEB2 protein functionality, accompanying milder clinical presentations.

Conclusion: Knowledge of the phenotypic spectrum of MWS and its correlation with the genotype will improve its detection rate and the prediction of its features, thus improving patient care.

Genet Med advance online publication 4 January 2018

Key Words: Hirschsprung; intellectual disability; management; Mowat-Wilson syndrome; ZEB2

\section{INTRODUCTION}

Mowat-Wilson syndrome (MWS) (OMIM \# 235730) is characterized by distinctive facial appearance in association with variable moderate-to-severe intellectual disability (ID), epilepsy, Hirschsprung disease (HSCR), and multiple congenital anomalies, including genital anomalies (in particular hypospadias), congenital heart disease, agenesis of the corpus callosum, and eye defects. MWS is caused by deleterious de novo heterozygous variations in the ZEB2 gene. The majority of variants lead to haploinsufficiency through premature stop codons or large deletions.

MWS was first delineated by Mowat et al. ${ }^{1}$ as a syndromic condition characterized by distinctive facial phenotype, ID, and Hirschsprung disease in four of six reported individuals. The causative genetic defect was mapped to chromosome 2q21-q23 based on cytogenetic deletions in two patients, ${ }^{1,2}$ and narrowed to heterozygous mutations of the ZEB2 gene by subsequent reports. ${ }^{3,4}$ In 2002 Zweier et al. $^{5}$ further delineated the phenotype of MWS with or without HSCR, invariably characterized by ZEB2 gene defects, and proposed that the condition be named Mowat-Wilson syndrome. More than 300 patients have been reported so $\mathrm{far}^{6-17}$ (additional reviewed articles are listed in Supplementary File S1 online).

We carried out a collaborative international study to further characterize and delineate the phenotype, natural history, and genotype-phenotype correlation of MWS.
We analyzed clinical data for 87 patients with a molecularly confirmed diagnosis of MWS, including 62 previously reported patients and 25 unpublished cases, and compared them with patients previously reported by other authors. We obtained these data through collaborations involving clinicians from various countries. Such primary data have never been collated from a large cohort of affected individuals.

In this article we present a comprehensive study of MWS features underlining a highly consistent phenotype for the disease, its genotype-phenotype correlations, and the phenotypic and clinical evolution taking place with age. The purpose of the article is to assist clinicians to identify the disease and to provide them with updated care recommendations for patient management. ${ }^{13}$

\section{MATERIALS AND METHODS}

Individuals affected by MWS and with a ZEB2 variation confirmed by appropriate methods (Supplementary File S2) were identified with the help of an international working group of collaborating clinicians and with the support of the Italian MWS Association. Through the respective clinicians, we invited them to fill in a highly detailed questionnaire. Informed consent for inclusion was obtained for all individuals involved in the study. Separate written informed consent was obtained for the publication of photographs. Approval for this study was granted by the Ethical Committee of Reggio Emilia.

\footnotetext{
Emilia, Modena, Italy; ${ }^{38}$ Danish Epilepsy Centre, Dianalund, Denmark; ${ }^{39}$ Institute for Regional Health Services, University of Southern Denmark, Odense, Denmark; ${ }^{40}$ Institute for Human Genetics, University Hospital Magdeburg, Magdeburg, Germany; ${ }^{41}$ Department of Pediatrics, Zealand University Hospital Roskilde, Roskilde, Denmark; ${ }^{42}$ Developmental Neurology Department, IRCCS Fondazione Istituto Neurologico “C. Besta," Milan, Italy; ${ }^{43}$ Medical Genetic Unit, AORN Cardarelli, Naples, Italy; ${ }^{44}$ Department of Pediatrics-Child Neurology Service, University Hospital Rijeka, Medical Faculty, University of Rijeka, Rijeka, Croatia; ${ }^{45}$ Department of Pediatrics, H. San Pedro, La Rioja, Logrono, Spain; ${ }^{46}$ Child Neuropsychiatry Unit, U.O.N.P.I.A ASST-Rhodense, Rho, Milan, Italy; ${ }^{47}$ Genetics Unit, St. Chiara Hospital, Trento, Italy; ${ }^{48}$ Department of Clinical Genetics, Leiden University Medical Center, Leiden, The Netherlands; ${ }^{49}$ Department of Pediatrics, IRCCS San Matteo, Pavia, Italy; ${ }^{50}$ Department of Medical Genetics, Gaetano Rummo Hospital, Benevento, Italy; ${ }^{51}$ Department of Pediatrics, Hospital S. Gerardo, University of Milano-Bicocca, Monza, Italy; ${ }^{52}$ Department of Pediatrics, ASST Lariana, Como, Italy; ${ }^{53}$ Department of Pediatrics, University of Torino, Torino, Italy; ${ }^{54}$ Department of Genetics, Wroclaw Medical University, Wroclaw, Poland; ${ }^{55}$ Clinical Genetics Unit, Division of Maternal Fetal Medicine, Department of Obstetrics and Gynecology, V. Buzzi Children's Hospital, Universita' degli Studi di Milano, Milan, Italy; ${ }^{56}$ Department of Pediatrics and Medical Sciences, "Vittorio Emanuele" Hospital, University of Catania, Catania, Italy; ${ }^{57}$ Department of Medical Genetics, Warsaw Medical University, Warsaw, Poland; ${ }^{58}$ Department of Pediatrics, University "La Sapienza," Rome, Italy; ${ }^{59}$ Department of Pediatrics, Ribeirão Preto Medical School, University of São Paulo, São Paulo, Brazil; ${ }^{60}$ Department of Genetics, Tours University Hospital, Tours, France; ${ }^{61}$ Department of Pediatrics, Eastern Virginia Medical School, Norfolk, Virginia, USA; ${ }^{62}$ Division of Medical Genetics and Metabolism, Children's Hospital of The King's Daughters, Norfolk, Virginia, USA; ${ }^{63}$ Department of Cell Biology, Erasmus University Medical Center, Rotterdam, The Netherlands; ${ }^{64}$ Institute of Genomic Medicine, Catholic University, Gemelli Hospital Foundation, Rome, Italy; ${ }^{65}$ Institute of Medical Genetics, University of Zurich, Schlieren-Zurich, Switzerland; ${ }^{66}$ Institute of Human Genetics, Friedrich-Alexander-Universität Erlangen-Nürnberg (FAU), Erlangen, Germany. Correspondence: Livia Garavelli (livia.garavelli@ausl.re.it)

The first three authors contributed equally to this work.
}

Submitted 7 September 2017; accepted 30 October 2017; advance online publication 4 January 2018. doi:10.1038/gim.2017.221 
Clinical and genetic data were transferred to an electronic database, and processed using the Statistical Package for the Social Sciences (SPSS, IBM, Chicago, IL), version 20.0. Group differences in phenotypes were compared using chi-square and Fisher's exact tests. Post hoc analysis was applied with Bonferroni correction for all multiple comparisons. $P<0.05$ was considered statistically significant.

\section{RESULTS}

A total of 87 patients are reported, ranging in age from 6 months to 36 years, including individuals not previously reported. The clinical diagnosis of MWS was confirmed by genetic testing in all of them. Patients originated from four continents and various ethnic backgrounds, although the vast majority were from Europe. The male-to-female ratio was 45:42. The mean age at the last clinical evaluation was 9 years and 10 months.

The main clinical features are presented in Table $\mathbf{1}$, in comparison with the literature. All numbers and percentages are related to the total number of patients that could be evaluated for a given feature. Figure 1 shows photographs of patients at various ages. Comprehensive clinical data are presented in Figure 2.

\section{Family history}

The average age of fathers and mothers at time of delivery was 34 years and 6 months and 31 years and 7 months respectively. No consanguinity was reported. Regarding two affected sisters presenting the same ZEB2 variant, germ-line mosaicism was considered because both parents had normal results on testing the mutation in blood. ${ }^{18}$ Germ-line mosaicism had already been considered in some previous reports. 19,20

\section{Birth history}

The average gestational age was 39 weeks, with a range between 33 and 43 . Weights at birth were within the normal range in both males and females. The average weight in males was $3,408 \mathrm{~g}$, with a range between $2,140 \mathrm{~g}$ and $4,680 \mathrm{~g}$; the average weight in females was $3,110 \mathrm{~g}$, with a range between $1,240 \mathrm{~g}$ and 4,100 g (Supplementary Table S1). The birth weight of all males was between the 2nd and 100th centiles for gestational age (mean 51st centile); the females' birth weight was between the 3rd and 100th centiles (mean 54th centile).

Length at birth was within the normal range for almost all the patients, both males and females. The average birth length in males was $49.9 \mathrm{~cm}$, with a range between 45.5 and $54 \mathrm{~cm}$ (range 2nd and 99th centiles, mean 44th). The average birth length in females was $49.2 \mathrm{~cm}$, with a range between 37 and $56 \mathrm{~cm}$ (range $<1$ st and 100th centiles, mean 53rd).

Although the average occipital frontal circumference at birth was within the normal range in both genders, the majority of patients had circumferences under the 50th centile, with mean values of 27th centile for males and 31st for females. In males, the mean was $33.5 \mathrm{~cm}$, with a range between 29.2 and $37 \mathrm{~cm}(<1 \mathrm{st}$ and 96th centiles); in females, the mean was $32.6 \mathrm{~cm}$, with a range between 27 and $35 \mathrm{~cm}$ $(<1$ st and 100th centiles).

\section{Head circumference and growth}

Microcephaly, i.e., occipital frontal circumference at least 2 SDs below the mean, was sometimes observed at birth, but more frequently tended to develop gradually throughout infancy but not all children were microcephalic (Figure 3a). Postnatally, microcephaly was noted in 5 of 14 patients (35.7\%, mean $47.7 \pm 2.6 \mathrm{~cm}$ ) in the age range from 0 to 5 years.

The majority of patients had a height below the 50th centile $(68 / 80,85 \%)$. Short stature was observed in 7 of 29 patients $(24.13 \%$, height $89.4 \pm 14.9 \mathrm{~cm})$ in the age range from 0 to 6 years, in 8 of 25 patients ( $32 \%$, height $125 \pm 13.4 \mathrm{~cm}$ ) in the age range from 6 to 12 years, and in 16 of 25 patients (64\%, height $150.8+16.8 \mathrm{~cm}$ ) over 12 years. Adult height in males

Table 1 Main clinical features in the present series and comparison with the literature

\begin{tabular}{|c|c|c|c|}
\hline & Our cohort $5,21,28$; & Literature $^{1-22 ; a, b}$ & Total $^{\mathrm{C}}$ \\
\hline Gender (male:female) & $45: 42$ & 138:119 & $183: 161$ \\
\hline Microcephaly & $55 / 81(67.9 \%)$ & $189 / 233(81.1 \%)$ & $244 / 314(77.7 \%)$ \\
\hline Hirschsprung disease & $26 / 85(30.6 \%)$ & $122 / 250(48.8 \%)$ & $148 / 335(44.2 \%)$ \\
\hline Constipation $^{d}$ & $37 / 85(43.6 \%)$ & $53 / 225(23.5 \%)$ & $90 / 310(29 \%)$ \\
\hline Congenital heart defects & $52 / 85(61.2 \%)$ & $141 / 247(57.1 \%)$ & 193/332 (58.1\%) \\
\hline Renal anomalies & $22 / 84(26.2 \%)$ & $37 / 149(24.8 \%)$ & $59 / 233(25.3 \%)$ \\
\hline Genital anomalies & $41 / 79(51.9 \%)$ & $64 / 165(38.8 \%)$ & $105 / 244(43 \%)$ \\
\hline Hypospadias & $28 / 45(62.2 \%)$ & $43 / 74(58.1 \%)$ & $71 / 119(59.7 \%)$ \\
\hline Cryptorchidism & $25 / 45(55.6 \%)$ & $26 / 78(33.3 \%)$ & $51 / 123(41.5 \%)$ \\
\hline Short stature & $31 / 79(39.2 \%)$ & $39 / 72(54.2 \%)$ & 70/151 (46.4\%) \\
\hline Structural eye anomalies & $8 / 83(9.6 \%)$ & $14 / 138(10.1 \%)$ & $22 / 221(9.9 \%)$ \\
\hline Cleft palate & $0 / 87(0.0 \%)$ & $4 / 140(2.9 \%)$ & $4 / 225(1.8 \%)$ \\
\hline
\end{tabular}

${ }^{a}$ Additional references in upplementary File $\mathbf{S 1}$. ${ }^{b}$ Patients with missense mutations in ZEB2 were not included. ${ }^{c}$ Only features for which we found precise information in the cited articles were considered. 'With absent or undetermined Hirschsprung disease. 


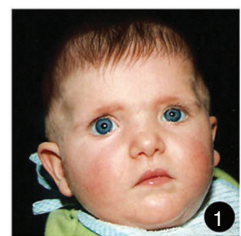

$\mathrm{P} 18$, infancy

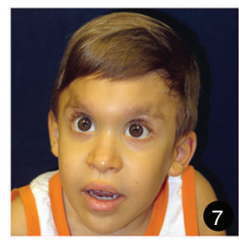

P89, 3 yr.

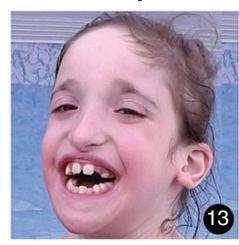

P54, 9 yr.

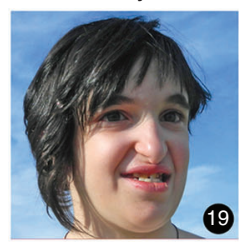

P23, 12 yr.

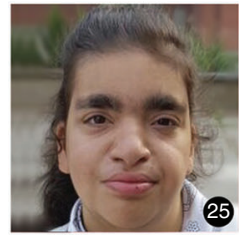

P58, 16 yr.

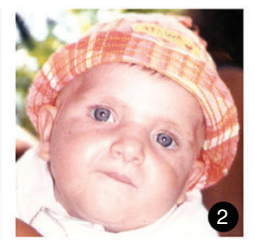

P18, infancy

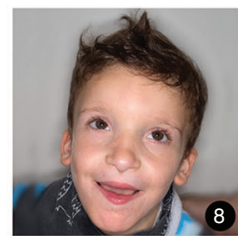

P10, 6 yr.

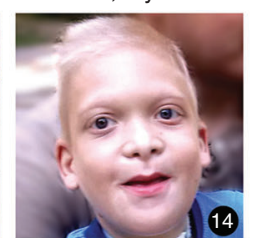

P80, 10 yr.

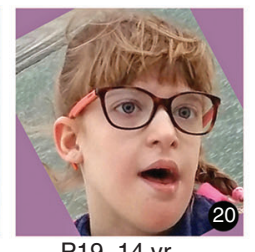

P19, 14 yr.

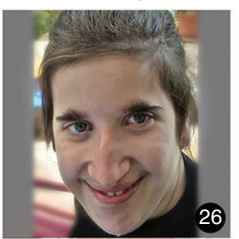

P55, 16 yr.

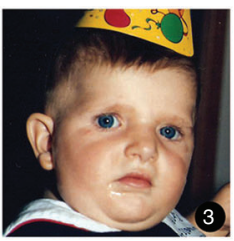

P18, infancy

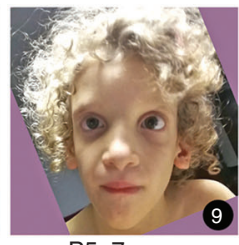

P5, 7 yr.

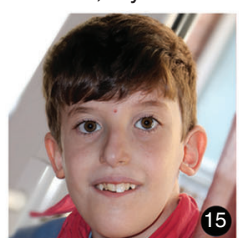

P31, 11 yr.

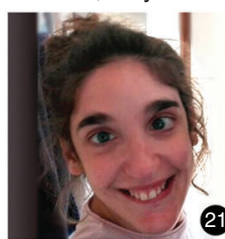

P29, 14 yr.

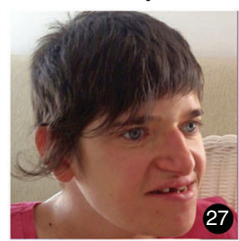

P18, 17 yr.

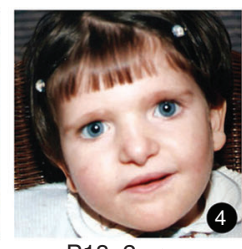

P18, 2 yr.

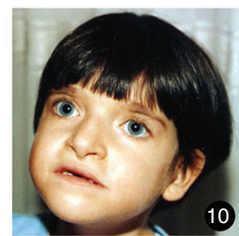

P18, 8 yr.

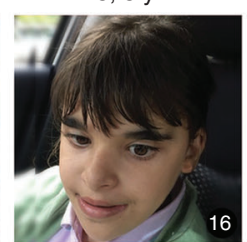

P8, 12 yr.

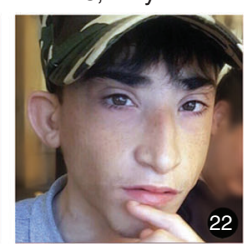

P40, 14 yr.

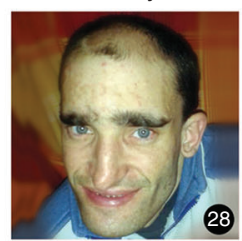

P32, 28 yr.

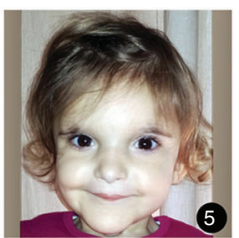

P35, 3 yr.

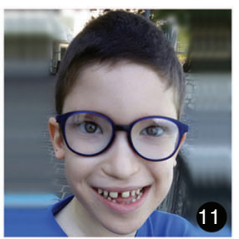

P27, 8 yr.

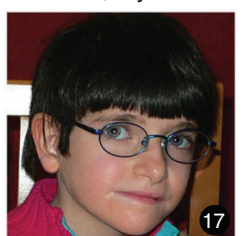

P18, 12 yr.

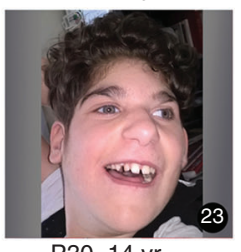

P30, 14 yr.

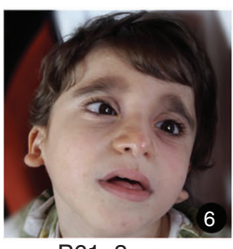

P61, 3 yr.

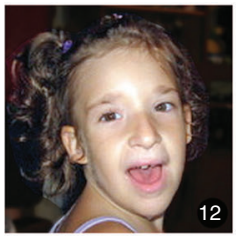

P1, 8 yr.

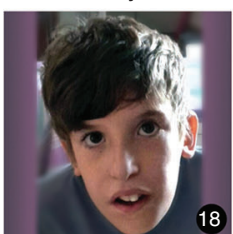

P31, 12 yr.

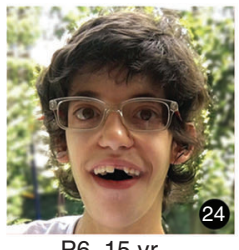

P6, 15 yr.

Figure 1 Photographs of patients with Mowat-Wilson syndrome at various ages. The numbered temporal sequence (left to right, top to bottom) shows how facial phenotype changes from infancy to adulthood. Age for each patient (P\#) is given in years.

varied between 155 and $185 \mathrm{~cm}(n=9$, mean $165.1 \mathrm{~cm})$ and in females between 146 and $155 \mathrm{~cm}(n=2$, mean $150.5 \mathrm{~cm})$.

Most patients were of slender build, with normal body proportions. Body weight was $<50$ th centile in 63 of 76 patients and $<3$ rd centile in 25 of 76 .

Overall, height (length) and weight were globally normal at birth, but often showed marked delay over time. Occipital frontal circumference, already under the 50th centile at birth, tended to fall progressively below normal values (Figure 3a).

\section{Facial phenotype}

All patients presented a distinctive facial appearance that renders the condition recognizable (Figure 1). A detailed list of the facial features is reported in Figure $\mathbf{2 a}$.

\section{Musculoskeletal anomalies}

Musculoskeletal anomalies were frequent and various. A detailed list of skeletal anomalies reported in our cohort can be found in Figure $\mathbf{2 b}$.

\section{Neurodevelopment, brain anomalies, and epilepsy}

MWS patients have moderate to severe ID. Hypotonia is often found in the first years of life and was detected in $79.1 \%$ of patients (Figure 2c). Developmental milestones such as sitting and walking were considerably delayed (Figure 2d): the mean age of sitting unsupported was 17.39 months, and the mean age of walking was 3 years and 9 months (range: 16 months to 11 years), although some individuals remain nonambulatory (33 of $86,38.3 \%$ ). A number of patients had a wide-based or ataxic-like gait, and sometimes held their arms up and flexed at the elbow, typical of individuals with Angelman syndrome. All milestones for fine motor skills were delayed. The majority of the oldest individuals (aged over 20 years) require help with dressing and other everyday activities. Speech rarely extends to more than a few words, with onset averaging at 4 years. Many patients have absence of speech (58 of 86 (67.4\%), 10 of them still under 4 years of age), but show receptive language skills and communicate successfully using alternative methods, such as augmentative and alternative communication (AAC). 
a

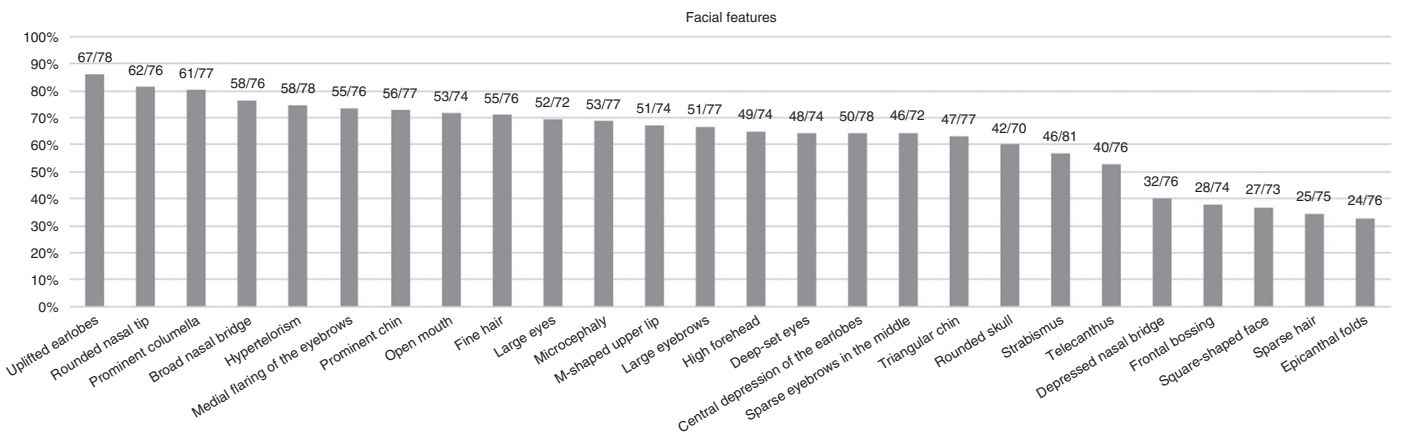

b

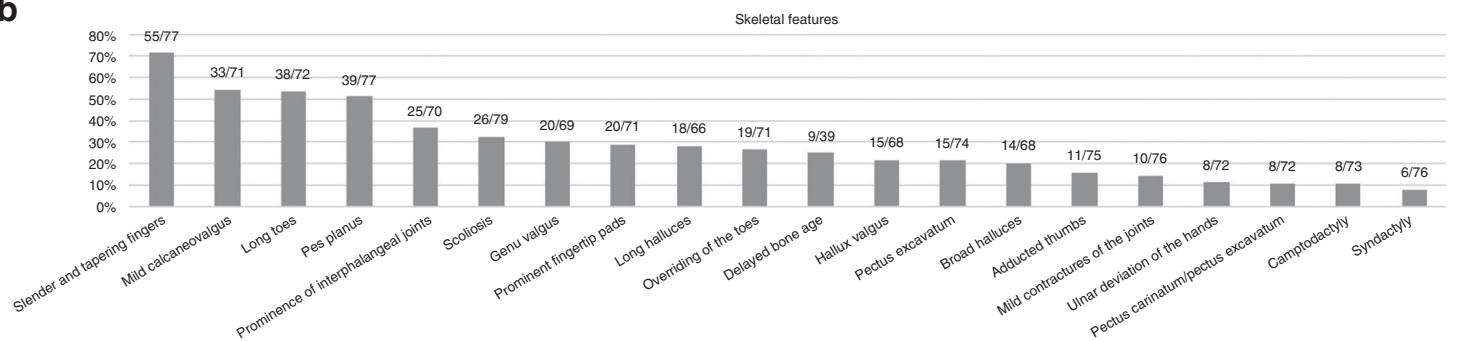

c
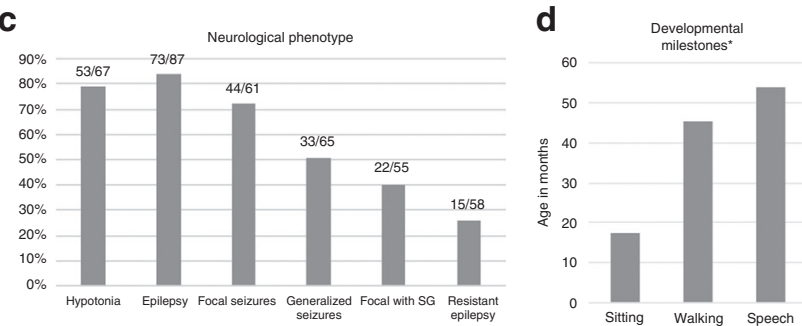

f

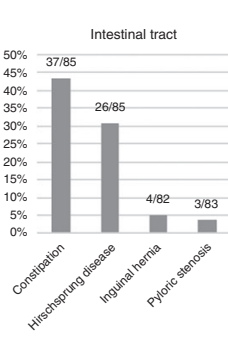

i

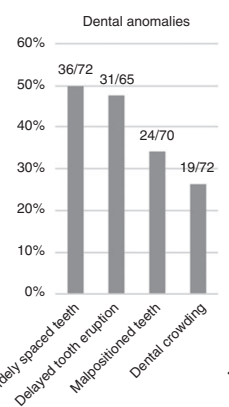

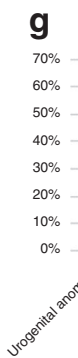

j

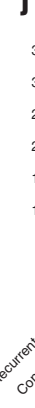

$35 \% 27$

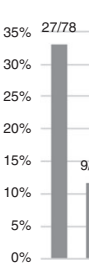

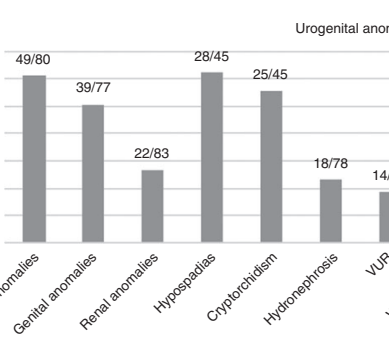

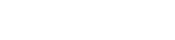

Other features

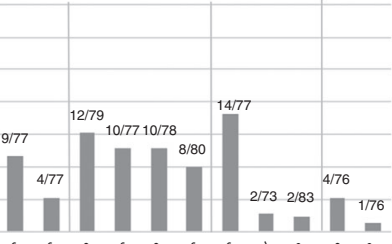

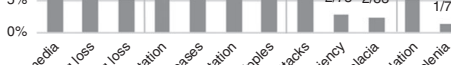

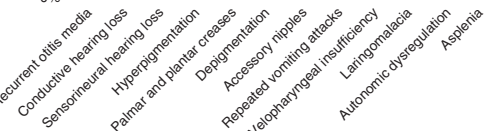

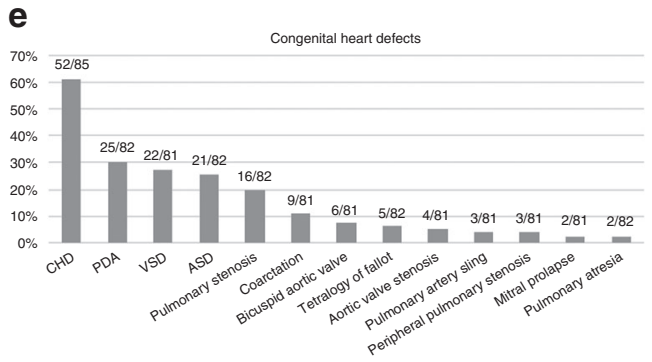

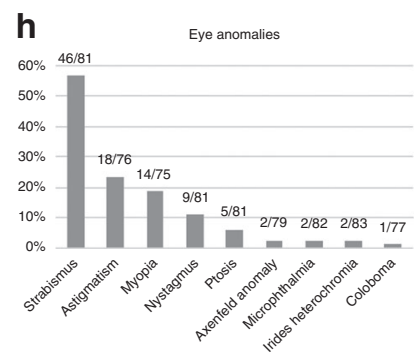

k

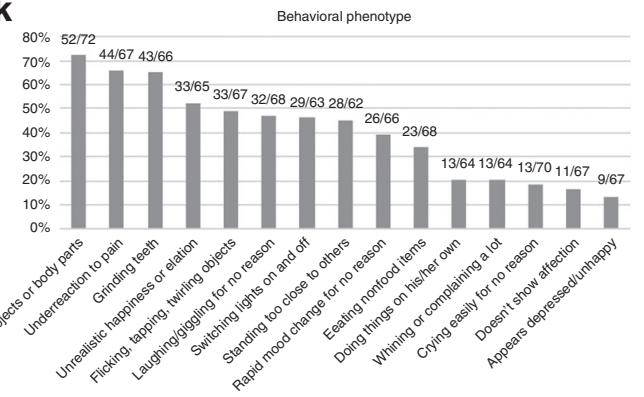

Figure 2 Clinical data for the presented patients. (a) Facial features, (b) skeletal features, (c) neurological phenotype, (d) developmental milestones, (e) congenital heart defects, (f) intestinal tract anomalies, (g) urogenital tract anomalies, (h) eye anomalies, (i) dental anomalies, (j) other features, (k) behavioral phenotype. *Normal developmental milestones range: sitting without support, 5-7 months; walking without support, 10-14 months; first words, 10-14 months. ASD, atrial septal defect; CHD, congenital heart disease; PDA, patent ductus arteriosus; SG, secondary generalization, VSD, ventricular septal defect; VUR, vesicoureteral reflux. 
a

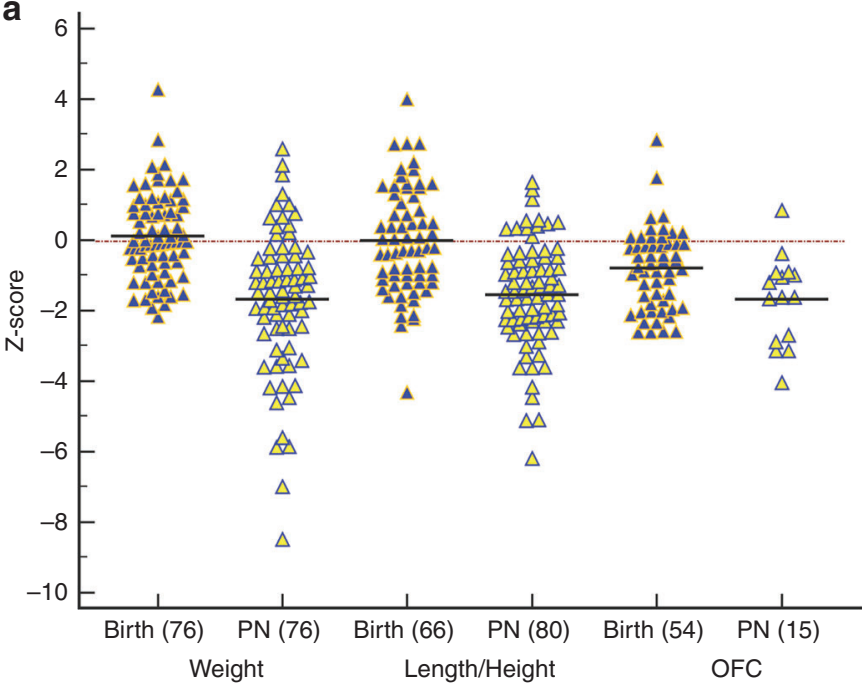

b

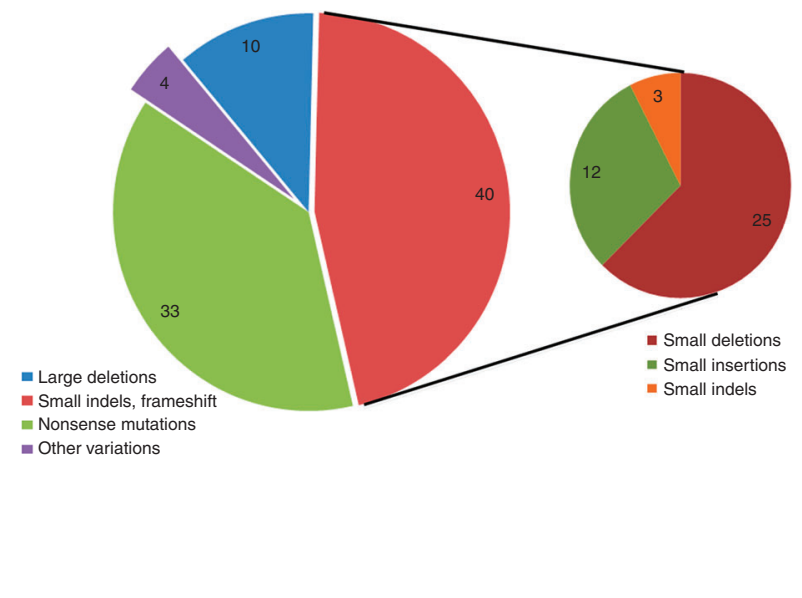

C

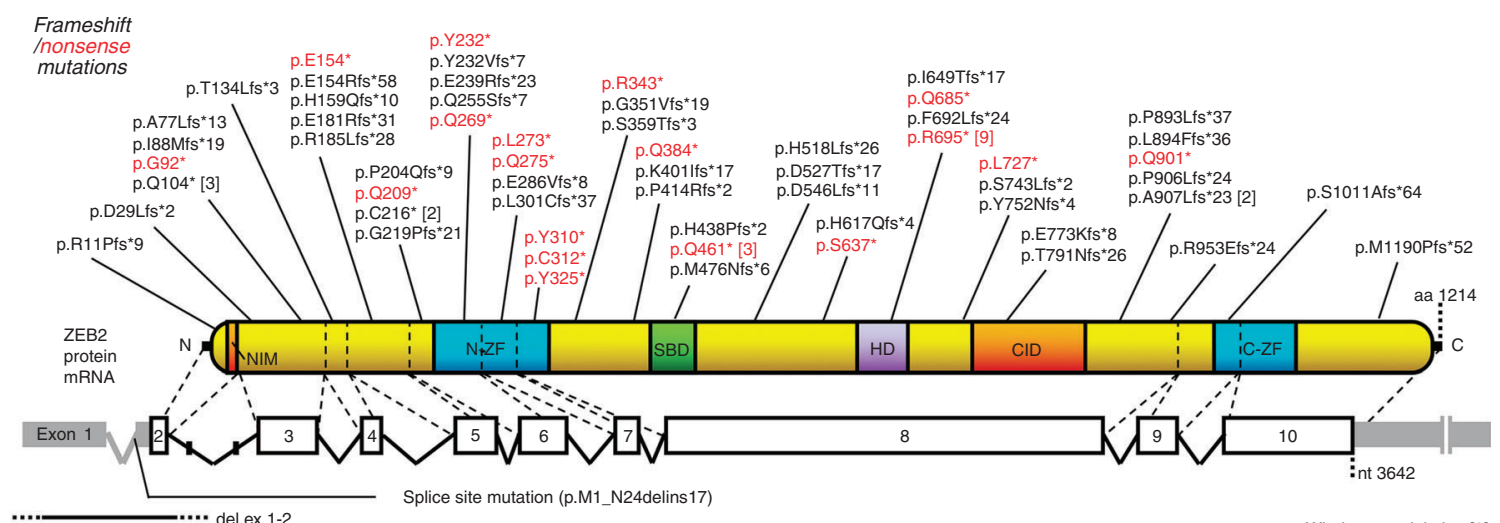

Whole-gene deletion [9]

Deletions del ex 8-9, in-frame (p.E307_G1023del)

del in $3^{\prime}$ UTR

d

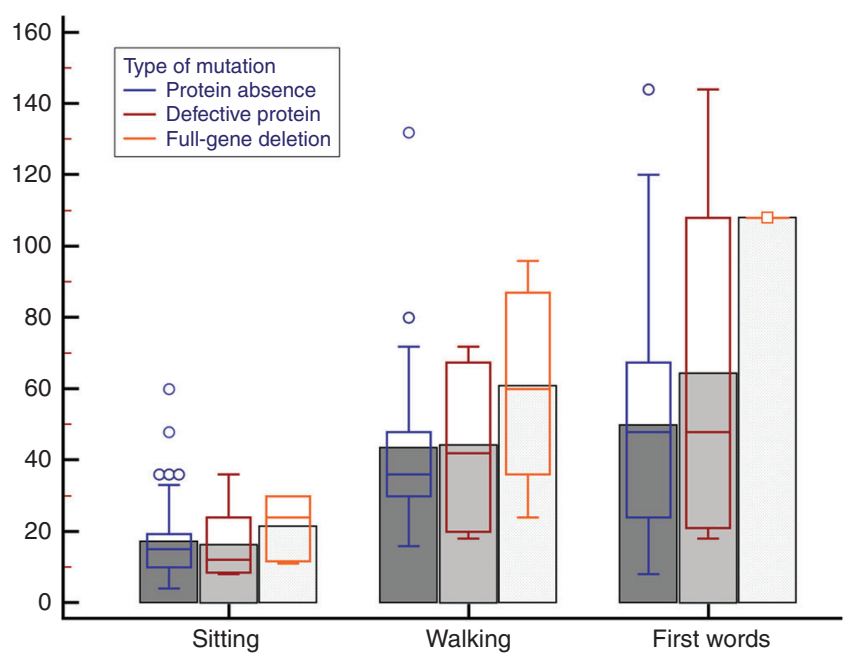

e

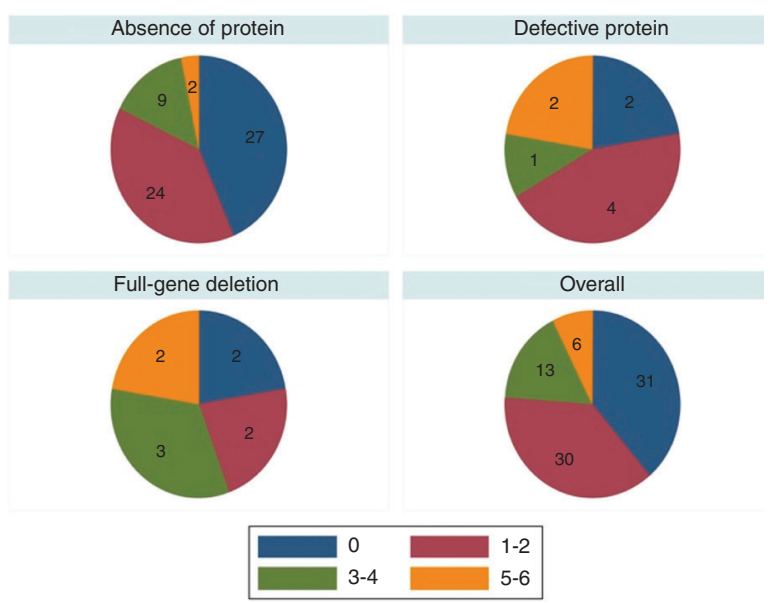

Figure 3 Evolution of some clinical features with age and genotype-phenotype correlations. (a) Growth parameters at birth and at last clinical evaluation postnatally. (b) Distribution of the different ZEB2 defects found in our cohort. (c) Position of genetic defects identified in our cohort on a schematic representation of the ZEB2 transcript and protein. (d) Correlation between developmental milestones and broad categories of the underlying heterozygous ZEB2 variants. Absence of protein (synthesis), defective protein (synthesis), and full-gene deletion all refer to the variant allele. Bars represent mean values, boxes represent median with interquartile (25th-75th percentile) range, whiskers represent range, and dots represents outliers. Only one patient in the full-gene deletion group developed speech, therefore no box plot representation was possible. (e) Number of cardiac anomalies in relation to broad categories of the underlying ZEB2 variants. mRNA, messenger RNA; OFC, occipital frontal circumference, PN postnatally. 
The neuroradiological phenotype in MWS patients, delineated recently, ${ }^{21}$ was characterized by anomalies of corpus callosum (79.6\% of cases), hippocampal abnormalities (77.8\%), enlargement of cerebral ventricles (68.5\%), and white matter abnormalities (reduction of thickness $40.7 \%$, localized signal alterations 22\%). We also observed large basal ganglia and cortical and cerebellar malformations.

It was observed that epilepsy was one of the most frequently detected features of MWS. Of the 87 patients in our cohort, $83.9 \%$ were found to have had seizures $(n=73 / 87)$ with a mean onset occurring at the age of 27.5 months. The most precocious onset in our cohort was registered at 1 month of age and the latest onset at 11 years of age. Our studies showed that $45 \%$ of patients have suffered fever-triggered seizures, $72.1 \%$ focal seizures, $50.8 \%$ generalized seizures, and $40 \%$ focal seizures with secondary generalization. In addition, $25.9 \%$ of patients had resistance to antiepileptic drugs (Figure 2c).

\section{Cardiac anomalies}

Congenital heart disease occurred in $60.5 \%$ of patients $(52 / 86)$ (Figure 2e). The most frequent cardiac anomalies $(>25 \%$ of cases) were not complex heart diseases and included patent ductus arteriosus, ventricular septal defect, and atrial septal defect. Pulmonary stenosis was present in almost 20\% of patients and aortic coarctation in more than $10 \%$. Other congenital heart defects with an incidence lower than $10 \%$ included bicuspid aortic valve, aortic valve stenosis, tetralogy of Fallot, pulmonary artery sling, proximal pulmonary artery stenosis, mitral valve prolapse, and pulmonary atresia.

\section{Gastrointestinal tract}

Constipation and Hirschsprung disease were frequent, occurring in $43.5 \%$ and $30.6 \%$ of patients, respectively (Figure 2f). Less common clinical features were inguinal hernia and pyloric stenosis.

\section{Urogenital/renal anomalies}

Urogenital anomalies were detected in 49 of 80 patients (61.25\%; Figure $\mathbf{2 g}$ ). The most frequent were hypospadias and cryptorchidism. Less common urogenital anomalies were webbed penis, microphallus, bifid scrotum, and vaginal septum. Renal anomalies included hydronephrosis, vesicoureteric reflux and, much more rarely, pelvic kidney.

\section{Eye anomalies}

The most frequent anomaly by far was strabismus $(56.8 \%$; Figure 2h). Quite common conditions were astigmatism, more common in females than in males (F:M = 2.6:1, $P=0.031)$, and myopia. Less frequent anomalies were nystagmus, palpebral ptosis, Axenfeld anomaly, microphthalmia, and irides heterochromia. Coloboma was present in only one patient.

\section{Tooth anomalies}

The two most frequent anomalies reported in our group were widely spaced teeth (50\%) and delayed tooth eruption (47.7\%)
(Figure 2i). Other less frequent but still fairly common anomalies were malpositioned teeth $(34.3 \%)$ and dental crowding (26.4\%).

\section{Other clinical features}

Recurrent otitis media was frequent, detected in $34.6 \%$ of patients. This feature must be taken into account whenever children present with fever because affected children tend to have a high pain threshold. It can contribute to conductive hearing loss, which in our cohort was present in 9 of 77 patients (11.6\%). Sensorineural hearing loss was less common (4/77, 5.2\%).

Repeated vomiting attacks were fairly common, with 14 of 77 patients detected (18.2\%).

Skin anomalies included hyperpigmentation (12/79, 15.2\%), depigmentation $(10 / 78,12.8 \%)$, and accessory nipples (8/80, 10\%).

Asplenia was fortunately rare (only 1 patient in our cohort), but it is important to investigate its presence, since it has been associated with serious infections (Figure 2j).

\section{Behavioral phenotype}

Some behavioral types were particularly frequent (present in over $60 \%$ of patients), including chewing or mouthing objects or body parts, underreaction to pain, and grinding of teeth. Other patterns, such as laughing for no obvious reason, fidgeting (flicking, tapping, and twirling of objects), unrealistic happiness or elation, switching lights on and off (more common in males than in females), rapid mood changes, standing close to others, and eating nonfood items, were also common (30-50\%; Figure 2k).

\section{Genotype}

All the genetic defects in our cohort were heterozygous, including chromosome deletions encompassing the ZEB2 locus on 2q21-q23 (detected by comparative genomic hybridization and multiplex ligation-dependent probe amplification), exon deletions (detected by multiplex ligationdependent probe amplification), and several different intragenic mutations (detected by direct sequencing).

Chromosome deletions (11.5\%) involved an entire ZEB2 allele or its first exons, and ranged in length from a few hundred kilobases to $16.7 \mathrm{Mb}$. Additionally, we found two intragenic deletions: one no larger than $11 \mathrm{~kb}$ and comprising exons $8-9$ and the other no larger than $28 \mathrm{~kb}$, encompassing exons 5-8, and leading to an altered reading frame downstream of the deletion.

The majority of pathogenic defects were intragenic mutations, either nonsense mutations (37.9\%), or small insertions/ deletions/indels (46\%) generating a frameshift and a premature stop codon (or, in one case, a longer protein with an altered C-terminus sequence).

Other variants included a deletion in the $3^{\prime}$ UTR region of $Z E B 2$, probably altering transcript synthesis or regulation, and an intronic variation altering the splice acceptor site of exon 2 , expected to generate a shorter protein with an altered 
$\mathrm{N}$-terminus sequence by similarity to the variant observed by Zweier et al. ${ }^{22}$

No patients with missense mutations were detected.

Genetic defects in our cohort are summarized in Supplementary Table S2 online and Figure 3b,c.

\section{DISCUSSION}

With more than 300 patients reported to date, MWS has become a well-known entity in clinical dysmorphology. Distinctive facial appearance is the most reliable clinical feature for recognizing this condition. Despite the fact that the facial gestalt is consistent with this diagnosis from an early age, a change in certain facial features with age has been reported. ${ }^{15}$ In childhood, earlobes seem to be uplifted, with a central depression, and eyebrows are large, medially flaring, and sparse in the middle. Facial features at this age also consist of a rounded skull, sparse and fine hair, puffy anterior neck, and excess skin on the rear of the neck. In childhood, the face is square with a high forehead, frontal bossing, epicanthal folds, hypertelorism, telecanthus, large and deepset eyes, strabismus, a broad nasal bridge, a rounded nasal tip, a prominent columella, an open mouth with an M-shaped upper lip, and a prominent and triangular chin. In addition, children with MWS tend to smile frequently. In children of school age, the face is longer, with a prominent jaw. The eyebrows are broad, usually exhibiting sparseness in the middle part.

Beginning in adolescence, the eyebrows often become heavier, broad, and horizontal; the nasal profile is more convex; the nasal tip lengthens, becomes more depressed, and overhangs the philtrum; the face tends to lengthen; and a long, pointed, or "chisel-shaped" chin may be observed.

The uplifted earlobes do not alter significantly over time apart from the central depression, which is less noticeable in adults, and this feature is useful in confirming suspicion of this condition.

What is most useful for clinicians to identify this condition is the overall facial gestalt, which changes over time. Figure 2a lists the most frequent facial features in our cohort in order of frequency. Two patients presented 25 of 26 facial features; the average number was 15.8. The three most frequent features (uplifted earlobes, a rounded nasal tip, and a prominent columella) were present together in 40 patients.

Before the delineation of a facial phenotype, HSCR was considered the hallmark of MWS. This led to reports of a high proportion of MWS patients with HSCR and underdiagnosis of patients with MWS but without HSCR. Afterward, as more MWS patients without HSCR were identified based on other features, the frequency of HSCR began to decrease. After reviewing the literature and analyzing our cohort's results, we concluded that the combined frequencies of HSCR and constipation were above $72 \%$. The difference in HSCR frequency in our cohort and the literature (30.6\% vs. $48.8 \%)$ reflects an enhanced knowledge of the clinical phenotype, which leads to the fact that patients are now not diagnosed on the basis of the presence of HSCR but rather on the basis of the facial gestalt. Because a considerable number of cases with severe constipation were not investigated by rectal biopsy due to risk of complications or lack of compliance, it is possible that the presence of HSCR is now underestimated. The data regarding the length of the aganglionic segment is not exhaustive in previously published cases, including our cohort. However, short and long segments have been reported both in males, in whom HSCR was more frequent, and in females.

Of note, although pulmonary artery sling with or without tracheal stenosis is quite rare (3.7\%), in the general population this cardiac anomaly is present in 1 of 17,000 school-aged children $(0.006 \%){ }^{23}$ This would make pulmonary artery sling more common in MWS patients, and we can therefore consider it a sign that may lead us to suspect MWS.

Ophthalmological findings have already been associated with MWS. ${ }^{24}$ Strabismus is the most common finding, followed by astigmatism, with a 2.6 times higher frequency in girls. Structural anomalies are rare, with Axenfeld anomaly and coloboma the most frequent.

Behavioral phenotypes in MWS have been delineated previously. ${ }^{25}$ According to our observations of the affected individuals, one feature requires special attention. Over $60 \%$ of patients (42 of 65) show underreaction to pain, which can be dangerous for these children. A recent study showed that the underreaction to pain results from a reduced responsivity to nociceptive stimulation rather than an inability to communicate discomfort. ${ }^{26}$

One of the major challenges posed by MWS is the recurrence of seizures. ${ }^{27}$ In 2013 Cordelli et al. ${ }^{28}$ hypothesized that a distinct electroclinical phenotype featuring focal and atypical absence seizures (which are often preceded by febrile seizures) and age-dependent electroencephalogram changes can be detected in the majority of patients with MWS.

Moreover, a recent paper $^{21}$ provided no evidence of a significant correlation between the brain malformation phenotype and the epilepsy phenotype in our cohort. This reinforces the hypothesis put forward by Cordelli et al..$^{29}$ that epilepsy in MWS has a genetic etiology.

The prevalence of MWS is low, which makes it still unknown to the majority of clinicians. This means that clinicians (pediatricians, neurologists, and/or clinical geneticists and genetic counselors) should be responsible for the coordination of patients' care and management. As the number of adult patients is increasing, the role of neurologists and clinical geneticists tends to become even more important. Multidisciplinary follow-up is essential and should consist of regular evaluations performed by different specialists; in the first place, pediatricians, neurologists, cardiologists, nephrologists, and gastroenterologists, especially during early childhood. Because there is no cure at this time, the goal of followup is improving quality of life. We strongly recommend AAC as a key point for MWS patient care. AAC has been recognized as a valuable tool to stimulate patients' communication skills, thus facilitating clinicians and parents in their 
Table 2 Summary of clinical follow-up and issues to be considered in an emergency situation Clinical evaluation/instrumental test Frequency

Pediatric assessment with measuring of growth parameters At time of diagnosis, every 6 months in the first 3 years of life, then yearly.

Neurological evaluation (including electroencephalogram when awake and At time of diagnosis, in cases of suspect symptoms for epileptic fits and then when asleep, brain magnetic resonance image, and polysomnography) and according to the evolution. Rehabilitation therapy is required and should be psychological treatment started as soon as possible. AAC is recommended.

Cardiological evaluation (including electrocardiogram and echocardiogram)

Pediatric gastroenterological evaluation anomalies that may be present.

Abdominal ultrasound (especially for presence/absence of spleen) In case of suspect HSCR in the first months of life and then at intervals determined by the clinical problems that may arise.

At time of diagnosis and then yearly or at intervals determined by clinical problems.

Ocular assessment At time of diagnosis then semiannually until the age of 6 or according to clinical problems.

Hearing evaluation At time of diagnosis and then at intervals determined by clinical problems.

Dental assessment

Orthopedic/physical medicine assessment

Surgical evaluation at infant age

Dietary assessment

Immunological pediatric evaluation

Spine $X$-rays Yearly from the age of 3 .

At time of diagnosis and then at intervals determined by clinical problems. Based on clinical evidence.

Based on clinical evidence.

In children with asplenia.

Based on clinical evidence.

Hematological tests: routine blood chemistry (blood count, renal and liver At time of diagnosis, then yearly.

function, glucose), APTT, PT-INR, immunological tests (IgG, IgA, IgM, and

lymphocyte subpopulations), ${ }^{a}$ thyroid function tests (thyroxine FT4, TSH

levels), thyroid autoantibodies (TPOAb, TGAb), antitransglutaminase

antibodies, IGF1, IGFBP3

\begin{tabular}{ll}
\hline Acute complication & Age range \\
\hline Repeated epileptic seizures & Especially in early childhood. \\
Aspiration pneumonia & All ages. \\
Heart failure in patients with congenital heart disease & Especially in neonatal and early childhood. \\
Gastrointestinal anomalies and complications of Hirschsprung disease (intestinal occlusion, enterocolitis) & Especially in neonatal and early childhood. \\
\hline Serious infections, sepsis & In children with asplenia. \\
Hematemesis/melena for bleeding from gastroesophageal reflux esophagitis & All ages. \\
\hline Acute otitis & All ages. \\
\hline
\end{tabular}

$\overline{\text { AAC, augmentative and alternative communication; APTT, activated partial thromboplastin time; HSCR, Hirschsprung disease; PT/INR, prothrombin time/international nor- }}$ malized ratio; TSH, thyroid-stimulating hormone.

${ }^{a}$ Also IgG subclass in children with asplenia.

compliance and determination of the behavioral phenotype. A detailed follow-up summary is reported in Table 2.

\section{Genotype-phenotype correlation}

We have grouped the genomic defects involving a single allele of ZEB2 into three major categories: deletions encompassing the whole gene, intragenic variants resulting in no protein synthesis/fast protein degradation, and intragenic variants resulting in synthesis of a defective protein.

The relatively small number of cases and the scarcity of functional data make it difficult to establish genotypephenotype correlations and assess their statistical significance, particularly where intragenic mutations are concerned.

Only a few clinical features show some level of significance for genotype-phenotype correlation (Supplementary Table S3), and they consistently involve comparing full-gene deletion with another genotypic category. These locus deletions often span several megabases of DNA and include other genes and long noncoding RNAs, such as the ZEB2antisense transcripts encoded in the $5^{\prime}$ portion of ZEB2 and involved in its transcriptional regulation. ${ }^{30}$ The variability and extent of these deletions may explain why they are usually associated with increased severity of some aspects, such as sitting age and onset of speech (Figure 3d). Full-gene deletion, or to a lesser extent the presence of a residual defective protein, also seems to negatively influence the number of cardiac defects, although their combined numbers are too small compared with cases of protein absence due to intragenic mutations (Figure 3e).

Transcripts from ZEB2 alleles with intragenic mutations are mostly predicted to undergo nonsense-mediated messenger RNA decay, or to generate very short and allegedly 
nonfunctional peptides. Here the variability of clinical presentations is likely due to a series of environmental and possibly genetic or epigenetic cofactors. Missense mutations of $Z E B 2$ are a rare occurrence and were found in none of our cases. They have been described in the literature in association with mild presentations sharing some similarity with MWS, ${ }^{31,32}$ although it is debatable whether most of these atypical cases should actually be classified as MWS.

However, in addition to patients with missense mutations, there are a few cases also expected to behave differently than typical haploinsufficiency. Notably, the least severely affected of our patients (P23) - a 12-year-old girl with typical facial features but mild to moderate ID, no seizures, absence of HSCR - has a frameshift mutation predicted to disrupt only part of the C-terminal zinc-finger domain: c.3031delA, p.(S1011Afs $\left.{ }^{\star} 64\right)$. Our preliminary studies, involving ZEB2 recombinant constructs expressed in a HEK293T cell line, indicate that this variant protein correctly localizes in the nucleus and retains some repressor ability on the E-cadherin promoter in a dual luciferase assay (unpublished data), possibly explaining the favorable clinical phenotype. Another patient (P34) with a very mild clinical presentation has a splicing defect that alters only the $\mathrm{N}$-terminal portion of the protein and abolishes the NuRD interacting motif, similarly to what was found in another patient. ${ }^{22}$ These observations seem to indicate that whenever a variant ZEB2 protein is predicted to preserve some of its functionality, we can expect a milder clinical presentation. The reverse might not always be the case, however; some mild cases have been found to have mutations abolishing ZEB2 protein function, suggesting the contribution of other genetic or environmental factors in shaping the MWS phenotype.

\section{Conclusions}

This paper analyzes data from the largest group of MWS patients reported to date.

Despite the variability of many features, MWS has a distinctive phenotype and is also genetically homogeneous, as all patients have a de novo mutation in a single gene, ZEB2, except for the rarest of cases in which a germ-line mosaicism has been proved in one of the parents.

The facial gestalt is easily recognizable, apart from the very rare patients with missense mutations. These should be discussed case by case as to whether a patient actually has MWS.

Since short stature is one of the most common features, we are currently collecting additional data to generate specific growth charts that will aid clinicians to improve the management and follow-up of children with MWS.

With the growing use of whole-exome sequencing for genes causing ID, we will undoubtedly identify ZEB2 mutations in patients who have a less obvious diagnosis of MWS. The study of these patients, as well as those with a milder phenotype and those with missense mutations, can help to define the boundaries of this condition. Better knowledge of the phenotypic spectrum of MWS and its genotype-phenotype correlations is likely to improve the detection rate of this condition and facilitate prediction of some MWS characteristics and a correct approach to some important features, such as epileptic seizures. This will hopefully aid in improving care for the patients and bringing much-needed support to their families.

\section{SUPPLEMENTARY MATERIAL}

Supplementary material is linked to the online version of the paper at http://www.nature.com/gim

\section{ACKNOWLEDGMENTS}

The financial support of Telethon Italy (grant GEP 14131) is gratefully acknowledged. The authors thank the Galliera Genetic Bank, member of the Telethon Genetic Biobank Network (project GTB12001), funded by Telethon Italy, and the Associazione Italiana Mowat Wilson ONLUS (AIMW), for their assistance and kind collaboration. We also thank all cooperating family members for contributing the medical data necessary for this study. In addition, we are grateful to Luca Valcavi for helping with the design and adaptation of the figures and tables. We also thank the photographer Marco Bonazzi, the genetic nurse Maria Claudia Menozzi, and the nurse Margherita Raucci for their excellent work. B.C. is a senior clinical investigator of the Scientific Research Fund-Flanders. Written consent for publication of the clinical pictures was obtained from the patients' parents.

\section{DISCLOSURE}

The authors declare no conflict of interest.

\section{REFERENCES}

1. Mowat DR, Croaker GD, Cass DT, et al. Hirschsprung disease, microcephaly, mental retardation, and characteristic facial features: delineation of a new syndrome and identification of a locus at chromosome 2q22-q23. J Med Genet. 1998;35:617-623.

2. Lurie IW, Supovitz KR, Rosenblum-Vos LS, Wulfsberg EA. Phenotypic variability of del(2) (q22-q23): report of a case with a review of the literature. Genet Couns. 1994;5:11-14.

3. Wakamatsu N, Yamada Y, Yamada K, et al. Mutations in SIP1, encoding Smad interacting protein-1, cause a form of Hirschsprung disease. Nat Genet. 2001;27:369-370.

4. Cacheux V, Dastot-Le Moal F, Kaariainen H, et al. Loss-of-function mutations in SIP1 Smad interacting protein 1 result in a syndromic Hirschsprung disease. Hum Mol Genet. 2001;10:1503-1510.

5. Zweier C, Albrecht B, Mitulla B, et al. "Mowat-Wilson" syndrome with and without Hirschsprung disease is a distinct, recognizable multiple congenital anomalies-mental retardation syndrome caused by mutations in the zinc finger homeo box 1B gene. Am J Med Genet. 2002;108:177-181.

6. Yamada K, Yamada $Y$, Nomura N, et al. Nonsense and frameshift mutations in ZFHX1B, encoding Smad-interacting protein 1, cause a complex developmental disorder with a great variety of clinical features. Am J Hum Genet. 2001;69:1178-1185.

7. Wilson M, Mowat D, Dastot-Le Moal F, et al. Further delineation of the phenotype associated with heterozygous mutations in ZFHX1B. Am J Med Genet A. 2003;119A:257-265.

8. Zweier C, Temple IK, Beemer F, et al. Characterisation of deletions of the ZFHX1B region and genotype-phenotype analysis in Mowat-Wilson syndrome. J Med Genet. 2003;40:601-605.

9. Cerruti Mainardi P, Pastore G, Zweier C, Rauch A. Mowat-Wilson syndrome and mutation in the zinc finger homeo box $1 \mathrm{~B}$ gene: a well defined clinical entity. J Med Genet. 2004;41:e16.

10. Ishihara N, Yamada K, Yamada Y, et al. Clinical and molecular analysis of Mowat-Wilson syndrome associated with ZFHX1B mutations and deletions at 2q22-q24.1. J Med Genet. 2004;41:387-393.

11. Garavelli L, Cerruti-Mainardi P, Virdis R, et al. Genitourinary anomalies in Mowat-Wilson syndrome with deletion/mutation in the zinc finger homeo box 1B gene (ZFHX1B). Report of three Italian cases with hypospadias and review. Horm Res. 2005;63:187-192.

12. Zweier C, Thiel CT, Dufke A, et al. Clinical and mutational spectrum of Mowat-Wilson syndrome. Eur J Med Genet. 2005;48:97-111. 
13. Adam MP, Schelley $S$, Gallagher $R$, et al. Clinical features and management issues in Mowat-Wilson syndrome. Am J Med Genet A. 2006;140:2730-2741.

14. Dastot-Le Moal F, Wilson M, Mowat D, Collot N, Niel F, Goossens M. ZFHX1B mutations in patients with Mowat-Wilson syndrome. Hum Mutat. 2007;28:313-321.

15. Garavelli L, Zollino M, Mainardi PC, et al. Mowat-Wilson syndrome: facial phenotype changing with age: study of 19 Italian patients and review of the literature. Am J Med Genet A. 2009;149A:417-426.

16. Yamada Y, Nomura N, Yamada K, et al. The spectrum of ZEB2 mutations causing the Mowat-Wilson syndrome in Japanese populations. Am J Med Genet A. 2014;164A:1899-1908.

17. Coyle D, Puri P. Hirschsprung's disease in children with Mowat-Wilson syndrome. Pediatr Surg Int. 2015;31:711-717.

18. Cecconi M, Forzano F, Garavelli L, et al. Recurrence of Mowat-Wilson syndrome in siblings with a novel mutation in the ZEB2 gene. Am J Med Genet A. 2008;146A:3095-3099.

19. McGaughran J, Sinnott S, Dastot-Le Moal F, et al. Recurrence of MowatWilson syndrome in siblings with the same proven mutation. Am J Med Genet A. 2005;137A:302-304.

20. Ohtsuka $M$, Oguni $H$, Ito $Y$, et al. Mowat-Wilson syndrome affecting 3 siblings. J Child Neurol. 2008;23:274-278.

21. Garavelli L, Ivanovski I, Caraffi SG, et al. Neuroimaging findings in Mowat-Wilson syndrome: a study of 54 patients. Genet Med. 2017;19: 691-700.

22. Zweier C, Horn D, Kraus C, Rauch A. Atypical ZFHX1B mutation associated with a mild Mowat-Wilson syndrome phenotype. Am J Med Genet A. 2006;140:869-872.

23. Yu JM, Liao CP, Ge S, et al. The prevalence and clinical impact of pulmonary artery sling on school-aged children: a large-scale screening study. Pediatr Pulmonol. 2008;43:656-661.

24. Bourchany A, Giurgea I, Thevenon J, et al. Clinical spectrum of eye malformations in four patients with Mowat-Wilson syndrome. Am J Med Genet A. 2015;167:1587-1592.

25. Evans E, Einfeld S, Mowat D, Taffe J, Tonge B, Wilson M. The behavioral phenotype of Mowat-Wilson syndrome. Am J Med Genet A. 2012;158A: 358-366.
26. Pradier B, Jeub M, Markert A, et al. Smad-interacting protein 1 affects acute and tonic, but not chronic pain. Eur J Pain. 2014;18:249-257.

27. Garavelli L, Mainardi PC. Mowat-Wilson syndrome. Orphanet J Rare Dis. 2007:2:42.

28. Cordelli DM, Garavelli L, Savasta S, et al. Epilepsy in Mowat-Wilson syndrome: delineation of the electroclinical phenotype. Am J Med Genet A. 2013;161A:273-284.

29. Cordelli DM, Pellicciari A, Kiriazopulos D, Franzoni E, Garavelli L. Epilepsy in Mowat-Wilson syndrome: is it a matter of GABA? Epilepsia. 2013;54: 1331-1332.

30. Beltran $M$, Puig I, Pena $C$, et al. A natural antisense transcript regulates Zeb2/Sip1 gene expression during Snail1-induced epithelial-mesenchymal transition. Genes Dev. 2008;22:756-769.

31. Ghoumid J, Drevillon L, Alavi-Naini SM, et al. ZEB2 zinc-finger missense mutations lead to hypomorphic alleles and a mild Mowat-Wilson syndrome. Hum Mol Genet. 2013;22:2652-2661.

32. Heinritz W, Zweier C, Froster UG, et al. A missense mutation in the ZFHX1B gene associated with an atypical Mowat-Wilson syndrome phenotype. Am J Med Genet A. 2006;140:1223-1227.

This work is licensed under a Creative Commons Attribution-NonCommercial-NoDerivs $\quad 4.0$

International License. The images or other third party material in this article are included in the article's Creative Commons license, unless indicated otherwise in the credit line; if the material is not included under the Creative Commons license, users will need to obtain permission from the license holder to reproduce the material. To view a copy of this license, visit http://creativecommons.org/licenses/ by-nc-nd/4.0/

(c) The Author(s) 2018 